\title{
Clinical value of SMI Combined with Low-Dose CT Scanning in differential diagnosis of Thyroid Lesions and Tumor Staging
}

\author{
Shao-wei Xue ${ }^{1}$, Yu-kun $\mathrm{Luo}^{2}, \mathrm{Zi}$-yu Jiao ${ }^{3}$, Lin Xü
}

\begin{abstract}
Objectives: To investigate the clinical value of Superb Microvascular Imaging (SMI) combined with low dose CT scanning in differential diagnosis of thyroid lesions and tumor staging.

Methods: A total of 120 patients with thyroid nodules admitted to the Chinese PLA General Hospital from January 2017 to July 2020 were selected. Paired design was adopted in this study. SMI and SMI combined with low-dose CT scanning were respectively carried out to these patients. The results were judged by two senior imaging physicians and two senior sonographers respectively. And t-test, $x^{2}$ test, Pearson correlation coefficient check and other methods were adopted to comparatively analyze the above two methods and the pathological results after operation or puncture.

Results: Compared with pathologic findings, the coincidence rate of SMI was $40 \%$, and the coincidence rate of SMI combined with low dose CT scanning was $84 \%$. The difference was significant $(\mathrm{p}=0.00)$; among the 120 thyroid nodule patients, 50 were diagnosed as malignant by pathological diagnosis, and 70 as benign; 27 malignant cases and 93 benign cases were detected by SMI; 48 malignant cases and 72 benign cases were detected by SMI combined with low dose CT scanning. The sensitivity and accuracy of the latter were significantly higher than those of the former, and the difference was statistically significant $(p=0.00)$; the enhancement, edge sharpness and homogeneity of SMI increased with the increase of tumor malignancy, showing positive correlation property.

Conclusion: SMI combined with low dose CT scanning has a higher diagnostic coincidence rate. Its sensitivity and accuracy are significantly superior. With the increase of tumor malignancy, the enhancement and unhomogeneity of SMI increase, and the edge is more blurred. That suggests: with the increase of tumor malignancy, neovascularization in the tumor is more obvious and more unevenly distributed; the increase of edge blur indicates more obvious tumor infiltration. The method has considerable clinical value for predicting the malignancy of tumors.
\end{abstract}

KEYWORDS: Superb Microvascular Imaging (SMI), Low-dose CT scanning, Thyroid nodules, Differential diagnosis.

doi: https://doi.org/10.12669/pjms.37.5.4144

How to cite this:

Xue SW, Luo YK, Jiao ZY, Xu L. Clinical value of SMI Combined with Low-Dose CT Scanning in differential diagnosis of Thyroid Lesions and Tumor Staging. Pak J Med Sci. 2021;37(5):1347-1352. doi: https://doi.org/10.12669/pjms.37.5.4144

This is an Open Access article distributed under the terms of the Creative Commons Attribution License (http://creativecommons.org/licenses/by/3.0), which permits unrestricted use, distribution, and reproduction in any medium, provided the original work is properly cited.

Correspondence:

Dr. Yu-kun Luo,

Department of Ultrasound Diagnosis,

The First Medical Center of Chinese PLA General Hospital,

Beijing 100853,

P.R. China.

Email: uba819@163.com

* Received for Publication:

* Revision Received:

* Revision Accepted: *

Pak J Med Sci September - October 2021
January 10, 2021

April 5, 2021

April 25, 2021

\section{INTRODUCTION}

Thyroid nodules are one of the most common space-occupying lesions in neck. The incidence of adults is about $5 \%,{ }^{1}$ and the incidence of females is higher than that of males. Although more than $90 \%$ of nodules are benign lesions, thyroid nodules are clinically important as they may represent approximately a thyroid cancer risk of $4.0 \%$ to $6.5 \%$. $^{2}$ Hence, careful assessment and exclusion must be 
carried out. ${ }^{3}$ At present, the diagnosis of thyroid nodules mainly depends on $\mathrm{CT}$, ultrasonography and other imageological examinations. ${ }^{4} \mathrm{CT}$ is highly sensitive to space-occupying lesions and can clearly show the boundary, internal morphological structure, size, cystic degeneration, calcification and so on of thyroid nodules, but CT is radioactive, and especially cephalic CT may do damage to the patients. ${ }^{5}$ Ultrasonic examination is non-invasive, simple and convenient, but it is difficult to clearly show the fine structure of the lesion. Superb Microvascular Imaging (SMI) is an advanced ultrasonic imaging technique. This technique, based on the fact that echo characteristics vary between human soft tissues, conducts intravenous injection of contrast agent to enhance the display of organs and lesions. Since the contrast agent microbubbles are small enough to pass microcirculation, SMI can display venules and arterioles, which is more conducive to judging the nature of pathological tissues. ${ }^{6}$ However, there are still limitations in the differential diagnosis of benign or malignant thyroid nodules. Especially when the nodules are of equal density, there is impact on the judgment results. ${ }^{7}$ The purpose of this study was to find a more advantageous method to determine the nature of thyroid nodules.

\section{METHODS}

Ethical Approval: The study was approved by the Institutional Ethics Committee of the first medical center of Chinese PLA General Hospital (No. S2019-178-02) in May 10, 2020, and written informed consent was obtained from all participants.

\section{Inclusion Criteria:}

1. Patients with thyroid nodules found on radiographic examination.

2. Patients with a definite pathological diagnosis (specimen sources: surgical specimens and puncture specimens). ${ }^{8}$

3. Conscious patients without mental disorders.
4. Patients and their families are willing and able to cooperate to complete the study and have good treatment compliance.

5. Patients aged between 30 and 60 years and generally in good condition.

\section{Exclusion Criteria:}

1. Patients who are not newly diagnosed.

2. Patients with non-thyroid tumors or other conditions that could affect the study results.

3. Patients with incomplete clinical or pathological data.

4. Patients suffering from mental illness or other cognitive impairment. All the subjects agreed to participate in the study and signed letters of informed consent.

A total of 120 patients with thyroid nodules admitted to the Chinese PLA General Hospital from January 2017 to July 2020 were selected and divided into malignant group and benign group after pathological examinations. Paired design was adopted in this study. There were 50 cases in malignant group, 19 males and 31 females aged $32 \sim 57$, average age: $46.83 \pm 8.20$, BMI: $(22.15 \pm 1.24)$ $\mathrm{kg} / \mathrm{m} 2$, maximum diameter of nodule: $4.21 \pm 0.76 \mathrm{~cm}$, course: $4.27 \pm 1.02$ years. There were 70 cases in benign group, 28 males and 42 females aged 30 58, average age: $46.39 \pm 8.79$, BMI: $(23.36 \pm 4.57) \mathrm{kg} / \mathrm{m} 2$, maximum diameter of nodule: $2.73 \pm 0.54 \mathrm{~cm}$, course: $2.15 \pm 0.88$ years. There is no significant difference between the two groups in general information (sex, age, BMI); there was significant difference $(p=0.00)$ in disease condition- the diameters of nodules in malignant group are larger than those in benign group (Table-I). Pathological results: malignant group: 26 cases of papillary carcinoma, 12 cases of follicular carcinoma, 8 cases of medullary carcinoma and four cases of undifferentiated carcinoma; benign group: nodular goiter 36 cases, adenoma 24 cases, Hashimoto's thyroiditis with nodule 5 cases, and other benign nodules 5 cases.

Method of CT scan Low dose CT scanning: The patient lies supine, head leaned back and neck fully

Table-I: Comparative analysis of general information between experimental group and control group $(\bar{X} \pm S)$.

\begin{tabular}{lcccc}
\hline Indicator & Malignant group $(50$ cases) & Benign group $(70$ cases $)$ & $t / \chi^{2}$ & $P$ \\
\hline Age (years old) & $46.83 \pm 8.20$ & $46.39 \pm 8.79$ & 0.28 & 0.78 \\
Male (cases, \%) & $19(38 \%)$ & $28(49 \%)$ & 0.05 & 0.82 \\
BMI $\left(\mathrm{kg} / \mathrm{m}^{2}\right)^{*}$ & $22.15 \pm 1.24$ & $23.36 \pm 4.57$ & 1.82 & 0.07 \\
Nodule diameter $(\mathrm{cm}) \Delta$ & $4.21 \pm 0.76$ & $2.73 \pm 0.54$ & 12.47 & 0.00 \\
Course (years)* & $3.27 \pm 1.02$ & $3.15 \pm 0.88$ & 0.68 & 0.49 \\
\hline
\end{tabular}

${ }^{*} \mathrm{P}>0.05 ; \Delta<0.05$. 
Table-II: Comparative analysis of coincidence rates of different examination methods with pathological results $(\bar{X} \pm S) n=50$.

\begin{tabular}{lccc}
\hline Method & Malignant & Pathological diagnosis & Coincidence rate $^{*}$ \\
\hline SMI & 20 & 50 & $40 \%$ \\
SMI combined with low dose CT scan & 42 & 50 & $84 \%$ \\
$\chi^{2}$ & & & 20.54 \\
$\mathrm{p}$ & & 0.00 \\
\hline
\end{tabular}

$* \mathrm{P}<0.05$.

exposed. Philips Ingenuity 64-layer SCT is used, ranging from $3 \sim 5 \mathrm{~cm}$ above the skull base to the level of thoracic entrance. Scanning parameters: $120 \mathrm{kV}, 90 \mathrm{mAs}$, thin layer scan, layer thickness and layer distance: 3-mm, pitch 0.87:1, tabletop speed $8.75 \mathrm{~mm} / \mathrm{r}$. Contrast agent for enhancement scan: iopromide, dose: $1.5 \mathrm{~mL} / \mathrm{kg}$. Delay time: $30 \mathrm{~s}, 60 \mathrm{~s}$. Two senior physicians evaluated and recorded the $\mathrm{CT}$ values of the nodules and judged the modes of CT enhancement: ${ }^{9} 1$. circular enhancement: the periphery of the nodule is circular enhancement, and the density is higher than that of the inside; 2 . homogeneous enhancement: the nodule is diffuse homogeneous enhancement; 3. nonhomogeneous enhancement: the nodule enhancement is inhomogeneously distributed; 4 . punctate enhancement: only local parts show sparse punctate enhancement inside the nodule; 5 . no enhancement: no obvious enhancement in the nodule.

Method of SMI: The patient lies supine, head leaned back and neck fully exposed. Ultrasound: Philips color Doppler ultrasound, linear array probe: L5-12, frequency: $12 \mathrm{MHz}$. First, routine ultrasonic scanning is performed, and the number, position, shape and size of nodules are recorded. Target nodules are selected for contrast-enhanced ultrasonography. The Bracco Sono Vuc contrast agent is taken and mixed with saline $(5 \mathrm{ml})$ to prepare microbubble suspension liquid. The liquid $(2 \mathrm{ml})$ is injected through the elbow vein. Rinse with $5.0 \mathrm{~mL}$ saline after injection. Press the timer key during contrast injection. Observe the nodule perfusion for at least 60s continuously. The results are evaluated by two senior sonographers, and the images are recorded in time. Judgement criteria: (1) enhancement degree: the echo intensity of peak lesion enhancement is divided into weak enhancement, equal enhancement and strong enhancement compared with the surrounding thyroid parenchyma; (2) enhancement homogeneity: the homogeneity of peak lesion enhancement is divided into homogeneous enhancement and nonhomogeneous enhancement (including local non-enhancement). ${ }^{10}$ Observation indicators: (1) The sensitivity, specificity and accuracy of SMI and SMI combined with low dose CT scanning in thyroid nodule differentiation (pathological results used as the diagnostic basis); (2) the coincidence rates between different examination methods and pathological results; (3) the correlations between different pathological types and SMI results.

Statistical Method: All data are processed with SPSS 20.0 software, and measurement data are expressed as $(\bar{X} \pm S)$. Intergroup data analysis is realized by t-test based on two groups of independent samples, and the rates are compared by $\chi^{2}$ test. The correlation is expressed by Pearson correlation coefficient, and $\mathrm{P}<0.05$ is statistically significant.

\section{RESULTS}

A comparative analysis of the two examination methods and the pathological results was conducted (Table-II). Among 50 malignant cases confirmed by pathological results, 20 cases were

Table-III: Comparative analysis of thyroid nodule differentiation results between SMI and SMI combined with low dose CT scanning.

\begin{tabular}{lcccccc}
\hline \multirow{2}{*}{ Pathological result } & \multicolumn{3}{c}{ SMI } & \multicolumn{3}{c}{ SMI combined with low dose CT scan } \\
\cline { 2 - 7 } & Malignant & Benign & Total & Malignant & Benign & Total \\
\hline Malignant & 20 & 30 & 50 & 42 & 8 & 50 \\
Benign & 7 & 63 & 70 & 6 & 64 & 70 \\
Total & 27 & 93 & 120 & 48 & 72 & 120 \\
\hline
\end{tabular}


Shao-wei Xue et al.

Table-IV: Analysis of sensitivity, specificity and accuracy of two groups.

\begin{tabular}{lccc}
\hline Method & Sensitivity $^{*}$ & Specificity & Accuracy $^{*}$ \\
\hline SMI & $40.00 \%(20 / 50)$ & $90.00 \%(63 / 70)$ & $69.17 \%(83 / 120)$ \\
SMI combined with low dose CT scan & $84.00 \%(42 / 50)$ & $91.42 \%(64 / 70)$ & $88.33 \%(106 / 120)$ \\
$X^{2}$ & 20.54 & 0.08 & 13.17 \\
P & 0.00 & 0.77 & 0,00 \\
\hline
\end{tabular}

$* \mathrm{P}<0.05$.

detected by SMI. The coincidence rate was $40 \%$; 42 cases were detected by SMI combined with low dose CT scanning. The coincidence rate was $84 \%$. The difference between the two groups was significant $(p=0.00)$, suggesting a higher coincidence rate of SMI combined with low dose CT scanning.

Of 120 thyroid nodule cases, 50 were pathologically diagnosed as malignant and 70 as benign. Among them, 27 malignant cases and 93 benign cases were detected by SMI; 48 malignant cases and 72 benign cases were detected by SMI combined with low dose CT scanning. The sensitivity and accuracy of the latter were significantly higher than those of the former, and the difference was statistically significant $(p=0.00)$. Table-III and Table-IV.

The correlation analysis between different pathological types and SMI results suggested: the enhancement, edge sharpness and homogeneity of SMI increased with the increase of tumor malignancy, showing positive correlation property (Table-V).

\section{DISCUSSION}

Thyroid nodules are masses of structural abnormality that occur in normal thyroid tissues due to certain causes. Thyroid nodules are the most common thyroid disease clinically. With the rising number of patients and the continuously improved examination methods, the detection rate of thyroid nodules is gradually increasing. Of all thyroid nodules, thyroid cancers account for less than $10 \%$, and most nodules are benign. Benign nodules generally have regular morphology and a clear boundary with the surrounding normal thyroid gland. Malignant nodules are mostly irregular in morphology, and have unclear boundaries with normal thyroid and surrounding structures, with blurred edges and hard texture. At present, the most commonly used imageological examination for the diagnosis of thyroid nodules are CT and ultrasound. Although routine ultrasonic examination is simple, fast and non-invasive, and can provide information for the diagnosis of thyroid nodules, there is subjectivity in determining the boundary, echo and calcification conditions. Conventional CT does harm to head and neck tissues.

Tumor vessels are the most important characteristic of tumor tissues. ${ }^{11}$ Research shows that compared with benign tumors; malignant tumors have different microvascular architecture. It is characterized by distortion and angiectasis of tumor vessels and structural disorder. ${ }^{12}$ Therefore, Zhou et al. ${ }^{13}$ believe that Superb Microvascular Imaging (SMI) is a more accurate method to display tumor microvessels. Adabi et al. ${ }^{14}$ believe that vascular network can provide valuable information about tumor angiogenesis. SMI has been found effective in the differentiation of breast lesions, thyroid nodules and liver lesions in human body. SMI is more effective than CDI/ PDI in detecting microvascular blood flow signals. For malignant nodules, SMI can more clearly describe the incompleteness of the peripheral microvascular system and the abnormalities of internal heterogeneous vascular system. Compared

Table-V: Correlation analysis between pathological types and SMI results.

\begin{tabular}{lccc}
\hline Type & Enhancement & Edge blur length & Nonhomogeneity \\
\hline Papillary & 0.32 & 0.35 & 0.23 \\
Follicular & 0.57 & 0.47 & 0.31 \\
Medullary carcinoma & 0.73 & 0.63 & 0.45 \\
Undifferentiated carcinoma & 0.81 & 0.74 & 0.47 \\
\hline
\end{tabular}

Pak J Med Sci September-October 2021 Vol. 37 No. $5 \quad$ www.pjms.org.pk 1350 
with conventional ultrasound, SMI can describe microvascular flow and vascular branches around and inside thyroid nodules in a more detailed and clear way. So it is a safe and low-cost scheme for distinguishing benign and malignant thyroid nodules. ${ }^{15}$

The main difference between malignant thyroid nodules and benign thyroid nodules is rapid angiogenesis. Blood vessels are usually richer and more disordered within malignant tissues. The study of Jiang et al. ${ }^{16}$ suggests: on contrast-enhanced ultrasound, most papillary thyroid carcinoma cases show heterogeneous low enhancement patterns, most nodular goiter patients show equal enhancement patterns, and adenoma patients show high enhancement patterns. Contrast-enhanced ultrasound is a practical and convenient method to identify malignant thyroid nodules. Hornung et al. ${ }^{17}$ believe that contrast-enhanced ultrasound is a highly sensitive method to detect microvascularization of thyroid cancer. Future studies should compare these findings with pathology of benign nodules to establish contrast-enhanced ultrasound as a standard diagnostic procedure for preoperative evaluation of suspicious thyroid nodules. The study of Carraro and Molinari suggests that. SMI has good consistency with biopsy results, so this method may be helpful for judging the degree of nodule differentiation in thyroid. ${ }^{18}$ Our study confirms: SMI and pathological types are correlated; the enhancement, edge blur length and inhomogeneity of SMI increase with the increase of tumor malignancy; thyroid nodules with high malignancy have abundant tumor vessels, which have great heteromorphism and infiltrate the surrounding tissues.

Nevertheless, contrast-enhanced ultrasound also has some defects. Mazzeo and his colleagues. ${ }^{19}$ believe that contrast-enhanced ultrasound may be helpful for some patients, while it may generate uncertain results in no enhancement patients. Ideally, another examination means is performed before a SMI examination is carried out, and then a comprehensive assessment result is offered. The findings of $\mathrm{Li}$ et al. ${ }^{20}$ show: the detection rates of conventional ultrasound and SMI decrease with the reduction of nodules, while CT is superior over ultrasound for small nodules. Traylor et al. ${ }^{21}$ believe that CT imaging is very important for preoperative evaluation of thyroid masses and thyroid cancers, while CT should be used as an important auxiliary means of SMI. ${ }^{22}$
The results of our study suggest that compared with pathologic findings, the coincidence rate of SMI was $40 \%$, and the coincidence rate of SMI combined with low dose CT scanning was $84 \%$. The difference was significant $(\mathrm{p}=0.00)$; among the 120 thyroid nodule patients, 50 were diagnosed as malignant by pathological diagnosis, and 70 as benign; 27 malignant cases and 93 benign cases were detected by SMI; 48 malignant cases and 72 benign cases were detected by SMI combined with low dose CT scanning. The sensitivity and accuracy of the latter were significantly higher than those of the former, and the difference was statistically significant $(p=0.00)$. Therefore, the combination of SMI and low dose CT scanning is more helpful for making up for the judgment of equal density nodules in SMI so as to improve the accuracy of diagnosis.

Limitations of this study: The combined method proposed by our study is still in the early stages of clinical application. Low-dose CT scanning and SMI are affected by many factors. In addition, the size of malignant sample is small. Further clinical exploration is necessary.

\section{CONCLUSION}

SMI combined with low dose CT scanning has significantly higher coincidence rate, sensitivity and accuracy. With the increase of tumor malignancy, the enhancement and inhomogeneity of SMI increase, and the edge is increasingly blurred. That indicates that with the increase of tumor malignancy, neovascularization in tumor is more obvious and more unevenly distributed. And the increase of edge blur indicates more obvious tumor infiltration. The findings are of certain clinical value for predicting the malignancy of tumor.

\section{Declaration of conflicting interest: None.}

Funding: None.

\section{REFERENCES}

1. Molinari F, Mantovani A, Deandrea $M$, Limone $P$, Garberoglio R, Suri JS. Characterization of single thyroid nodules by contrast-enhanced 3-D ultrasound. Ultrasound Med Biol. 2010;36(10):1616-1625. doi: 10.1016/j. ultrasmedbio.2010.07.011

2. Zamora EA, Khare S, Cassaro S. Thyroid Nodule. In: StatPearls. Treasure Island (FL): StatPearls Publishing; August 10, 2020.

3. Van Vlaenderen J, Logghe K, Schiettecatte E, Vermeersch $\mathrm{H}$, Huvenne W, De Waele K, et al. A synchronous papillary and follicular thyroid carcinoma presenting as a large toxic nodule in a female adolescent. Int J Pediatr Endocrinol. 2020;2020:14. doi: 10.1186/s13633-020-00084-4 
4. Giansante L, Martins JC, Nersissian DY, Kiers KC, Kay FU, Sawamura MVY, et al. Gebrim EMMS, Costa PR. Organ doses evaluation for chest computed tomography procedures with TL dosimeters: Comparison with Monte Carlo simulations. J Appl Clin Med Phys. 2019;20(1):308 320. doi: $10.1002 / \mathrm{acm} 2.12505$

5. Perincek G, Avci S, Celtikci P. Retrosternal Goiter: A couple of classification methods with computed tomograpy findings. Pak J Med Sci. 2018;34(6):1494-1497. doi: 10.12669 / pjms.346.15932

6. Ghavami S, Bayat M, Fatemi M, Alizad A. Quantification of Morphological Features in Non-Contrast-Enhanced Ultrasound Microvasculature Imaging. IEEE Access. 2020;8:18925-18937. doi: 10.1109/ ACCESS.2020.2968292

7. Yeom JA, Roh J, Jeong YJ, Lee JC, Kim HY, Suh YJ, et al. Ultra-Low-Dose Neck CT With Low-Dose Contrast Material for Preoperative Staging of Thyroid Cancer: Image Quality and Diagnostic Performance. AJR Am J Roentgenol 2019;212(4):748-754. doi: 10.2214/AJR.18.20334

8. Pemayun TG. Current Diagnosis and Management of Thyroid Nodules. Acta Med Indones. 2016;48(3):247-257.

9. Hoang JK, Branstetter BF 4th, Gafton AR, Lee WK, Glastonbury CM. Imaging of thyroid carcinoma with CT and MRI: Approaches to common scenarios. Cancer Imaging. 2013;13(1):128-139. doi: 10.1102/14707330.2013.0013

10. Tian C, Wang Z, Hou X, Wang C. The diagnostic accuracy of superb microvascular imaging in distinguishing thyroid nodules: A protocol for systematic review and meta analysis. Medicine (Baltimore). 2020;99(40):e22350. doi: 10.1097/MD.0000000000022350

11. Johnson RH, Chien FL, Bleyer A. Incidence of breast cancer with distant involvement among women in the United States, 1976 to 2009. Published correction appears in JAMA. 2013;309(12):1229 JAMA. 2013;309(8):800-805. doi: 10.1001/jama.2013.776

12. Yongfeng Z, Ping Z, Hong P, Wengang L, Yan Z. Superb microvascular imaging compared with contrast-enhanced ultrasound to assess microvessels in thyroid nodules. J Med Ultrason (2001). 2020;47(2):287-297. doi: 10.1007/ s10396-020-01011-Z

13. Zhou J, Luo Y, Ma BY, Ling WW, Zhu XL. Contrastenhanced ultrasound diagnosis of hepatic metastasis of concurrent medullary-papillary thyroid carcinoma: A case report. Medicine (Baltimore). 2017;96(50):e9065. doi: 10.1097/MD.0000000000009065

14. Adabi S, Ghavami S, Fatemi M, Alizad A. Non-Local Based Denoising Framework for In Vivo ContrastFree Ultrasound Microvessel Imaging. Sensors (Basel). 2019;19(2):245. doi: 10.3390/s19020245
15. Lu R, Meng $Y$, Zhang $Y$, Zhao W, Wang X, Jin M, et al. Superb microvascular imaging (SMI) compared with conventional ultrasound for evaluating thyroid nodules. BMC Med Imaging. 2017;17(1):65. doi: 10.1186/s12880017-0241-5

16. Jiang J, Shang $\mathrm{X}$, Zhang $\mathrm{H}, \mathrm{Ma} \mathrm{W}, \mathrm{Xu} \mathrm{Y}$, Zhou $\mathrm{Q}$, et al. Correlation between maximum intensity and microvessel density for differentiation of malignant from benign thyroid nodules on contrast-enhanced sonography. J Ultrasound Med. 2014;33(7):1257-1263. doi: 10.7863/ ultra.33.7.1257

17. Hornung M,JungEM,Georgieva M,SchlittHJ,Stroszczynski C, Agha A. Detection of microvascularization of thyroid carcinomas using linear high resolution contrast-enhanced ultrasonography (CEUS). Clin Hemorheol Microcirc. 2012;52(2-4):197-203. doi: 10.3233/CH-2012-1597

18. Carraro R, Molinari F, Deandrea M, Garberoglio R, Suri JS. Characterization of thyroid nodules by 3-D contrastenhanced ultrasound imaging. Annu Int Conf IEEE Eng Med Biol Soc. 2008;2008:2229-2232. doi: 10.1109/ IEMBS.2008.4649639

19. Mazzeo S, Caramella D, Marcocci C, Lonzi S, Cambi L, Miccoli P, et al. Contrast-enhanced color Doppler ultrasonography in suspected parathyroid lesions. Acta Radiol. 2000;41(5):412-416. doi: $10.1080 / 028418500127345820$

20. Li JW, Chang C, Chen JY, Shi ZT, Chen M. Nodule Size Effect on Diagnostic Performance of Ultrasonography and Computed Tomography for Papillary Thyroid Carcinoma. Curr Med Imaging Rev. 2019;15(5):489-495. doi: 10.2174/1 573405614666180425142141

21. Traylor KS. Computed Tomography and MR Imaging of Thyroid Disease. Radiol Clin North Am. 2020;58(6):1059 1070. doi: 10.1016/j.rcl.2020.07.004

22. Jainulabdeen $\mathrm{T}$, Ramaswamy B, Devaraja $\mathrm{K}$, Paruthikunnan SM, Bhandarkar AM. Preoperative Staging of Differentiated Thyroid Carcinomas: Comparison of USG and CT with Intraoperative Findings and Histopathology. Indian J Otolaryngol Head Neck Surg. 2019;71(3):327-333. doi: 10.1007/s12070-019-01663-5

\section{Authors' Contributions:}

SWX \& YKL: Designed this study and prepared this manuscript, and are responsible and accountable for the accuracy or integrity of the work.

LX; \& ZYJ: Collected and analyzed clinical data and significantly revised this manuscript.

Authors:

1. Shao-wei Xue,

Department of Ultrasound Diagnosis,

2. Yu-kun Luo,

Department of Ultrasound Diagnosis,

3. Zi-yu Jiao,

Department of Ultrasound Diagnosis,

4. Lin Xu,

Department of Radiology,

1-4: The First Medical Center of Chinese PLA General Hospital, Beijing 100853

P. R. China. 